\title{
The preventive effect of breast-feeding for longer than 6 months on early pubertal development among children aged 7-9 years in Korea
}

\author{
Hye Ah Lee ${ }^{1}$, Young Ju Kim ${ }^{2}$, Hwayoung Lee ${ }^{3}$, Hye Sun Gwak ${ }^{4}$, Young Sun Hong ${ }^{5}$, \\ Hae Soon $\mathrm{Kim}^{6}$, Eun Ae Park ${ }^{6}$, Su Jin $\mathrm{Cho}^{6}$, Eun Hee Ha ${ }^{1}$ and Hyesook Park ${ }^{1, *}$ \\ 'Department of Preventive Medicine, School of Medicine, Ewha Womans University, 1071 Anyangcheon-ro, \\ Yangcheon-ku, Seoul 158-710, Republic of Korea: ${ }^{2}$ Obstetrics and Gynecology, School of Medicine, Ewha \\ Womans University, Seoul, Republic of Korea: ${ }^{3}$ Anatomy, School of Medicine, Ewha Womans University, Seoul, \\ Republic of Korea: ${ }^{4}$ Colleage of Pharmacy, Ewha Womans University, Seoul, Republic of Korea: ${ }^{5}$ Internal Medicine, \\ School of Medicine, Ewha Womans University, Seoul, Republic of Korea: ${ }^{6}$ Department of Pediatrics, School of \\ Medicine, Ewha Womans University, Seoul, Republic of Korea
}

Submitted 14 April 2014: Final revision received 9 January 2015: Accepted 14 January 2015: First published online 6 March 2015

\begin{abstract}
Objective: The present study was performed to investigate whether breast-feeding is associated with early pubertal development among children 7-9 years old in Korea.

Design: Children were divided into those who did and did not receive breastfeeding for 6 months or longer in accordance with the recommendations of the WHO. Pubertal status was determined by clinical examination using Tanner staging.

Setting: Prospective observational study.

Subjects: We conducted a follow-up study of children aged 7-9 years in 2011 who had taken part in the Ewha Birth \& Growth Cohort study.

Results: Fifty (22.8\%) of the total of 219 children were in early puberty, with the proportion being slightly higher for girls (24.1\%) than boys (21.4\%). Children who had entered early puberty were taller, weighed more and had a higher concentration of insulin-like growth factor 1 . Moreover, the change in weight $Z$-score from birth to follow-up was significantly lower in children who were breast-fed than in those who were not (weight $Z$-score change: 0.32 (SD 1.59) $v$. 0.77 (SD 1.61), respectively, $P=0.04$ ). Comparison of breast-feeding by puberty status indicated a preventive association with early puberty in children who were breast-fed for 6 months or longer ( OR $=0.37$; $95 \%$ CI $0 \cdot 18,0 \cdot 74)$. This association remained significant after adjustment for relevant covariates.

Conclusions: These results demonstrate a beneficial association between breastfeeding and early pubertal development, especially in those breast-fed for 6 months or longer. The study suggests that interventions would need to start early in life to prevent early pubertal development.
\end{abstract}

\author{
Keywords \\ Breast-feeding \\ Cohort study \\ Early puberty development
}

The incidence of precocious puberty has increased consistently over the past several decades in the Republic of Korea and has become a major public health problem. The age of menarche occurs earlier than it did several decades ago, as reported in the 2005 Korean National Health and Nutrition Survey, which estimated that the average age at menarche decreased from 16.9 years in women born between 1920 and 1925 to $13 \cdot 1$ years in women born between 1986 and $1995^{(1)}$. Although the reasons underlying the forward shift in the average age of puberty onset are not well understood, the early development of puberty could lead to various psychological and social problems because physical development is not in line with cognitive development ${ }^{(2)}$. It is also thought to be a potential risk factor for polycystic ovary syndrome, breast cancer and $\mathrm{CVD}^{(3,4)}$.

Puberty is a complex developmental process that depends on a number of factors, including prenatal, behavioural and environmental factors, as well as genetic heritability. Interestingly, some studies suggest that nutrition during infancy, especially breast-feeding, may have a favourable effect on the timing of pubertal development ${ }^{(5,6)}$. Breast-feeding is 
well known for its positive impact on infant growth and child health. Additionally, the WHO recommends breast-feeding for the first 6 months to achieve optimal growth $^{(7)}$. Nutrition during infancy is directly associated with weight gain, which also has a dominant effect on the development of puberty. Although breast-feeding has been recommended to prevent early puberty, there are conflicting opinions regarding whether breast-feeding is related to a decrease in age of puberty onset ${ }^{(8-11)}$. Recently, a study reported that an additional month of breast-feeding decreases the risk of earlier menarche ${ }^{(5)}$, but that study did not consider postnatal growth.

Therefore, we investigated the association between breast-feeding and early pubertal development in Korean children aged 7-9 years considering the growth change from birth to age at follow-up.

\section{Methods}

\section{Sample}

We conducted a follow-up study in 2011 of 7-9-year-old subjects who had participated in the Ewha Birth \& Growth Cohort study, a prospective cohort that was established from 2001 to 2006. That study followed a single hospitalbased cohort composed of offspring and their mothers, who were recruited between 24 and 28 weeks of pregnancy during prenatal care visits at Ewha Womans University Mokdong Hospital, Seoul, Republic of Korea. This is a tertiary general hospital and one of the largest facilities (857 beds; an average of $~ 2700$ out-patients daily) in the western area of central Seoul. During the recruitment period from August 2001 to August 2006, a total of 940 pregnant women participated in the study. From this cohort, 664 children were eligible for the present study. Only 344 subjects were contacted, and 260 of them participated in the 2011 follow-up programme. The cohort composition and methodology are described in a previous study ${ }^{(12)}$

The follow-up programme of the Ewha Birth \& Growth Cohort study has been conducted annually since 2005, when the participating children reached 3, 5, 7, 8 and 9 years of age. In 2011, we performed the first follow-up with 9-year-old subjects and conducted clinical assessments of puberty. We contacted a total of 344 parents of children 7-9 years old by telephone. They were informed of the follow-up, and the subjects visited the hospital. For the present study, data were collected from July to August 2011. A total of 260 subjects $(75.6 \%)$ participated in the 2011 follow-up programme with consent from the children and their parents or guardians. The characteristics of the participants compared with those of non-participants were as follows: maternal age (years) $=31.43(\mathrm{sD} 3.64)$ v. 31.18 (SD 3.57) and birth weight $(\mathrm{kg})=3.20(\mathrm{SD} 0.52)$ v. 3.18 (SD 0.53), respectively; these differences were not significant. Detailed follow-up data were obtained from the participants through a questionnaire and by blood and urine sampling.

Subjects were excluded if a blood sample was not obtained ( $n$ 1), data for breast-feeding were missing ( $n$ 10), or if they were small or large for gestational age ( $n 28$ and $n 2$, respectively). Complete data were available for 103 boys ( $47.0 \%$ ) and 116 girls (53.0\%), a total of 219 , consisting of eighty-two subjects at 7 years old (37.4\%), fifty-seven at 8 years old $(26.0 \%)$ and eighty at 9 years old $(36.5 \%)$. The study protocol was approved by the Institutional Review Board of Ewha Womans University Hospital.

\section{Data collection}

Data regarding the occurrence and total duration of breastfeeding were gathered from parents or guardians using questionnaires. According to WHO recommendations ${ }^{(7)}$, infants should be exclusively breast-fed for the first 6 months to achieve optimal growth. In our study, we collected responses to the question: 'How long did you breast-feed your baby following birth?' We did not ask specifically about 'exclusive breast-feeding' because it is difficult to define. We classified the subjects into two groups: those who were breast-fed for 6 months or longer (any breast-feeding, $n$ 113) and those who were not ( $n$ 106). Additionally, the 6-month criterion was a statistical median point of breast-feeding duration in our data. We repeatedly collected breast-feeding data by questionnaire at 12 months, 18 months and during the present follow-up study. The questionnaires at 12 and 18 months were returned by mail. The return rates were $48.8 \%$ at 12 months and $41.0 \%$ at 18 months. As a result of followup loss, data from only seventy-five subjects (single response at 12 months: $n$ 23; single response at 18 months: $n$ 21; response at 12 and 18 months: $n$ 31) were used. When the two groups were classified by report of 6 months or more of breast-feeding or not, the kappa value was 0.9 . We used parents' recall of earlier breastfeeding collected when the children were 7-9 years of age (follow-up year, 2011) because some data from 12 and 18 months of age were missing.

We collected data regarding possible risk factors including birth weight, gestational age and maternal age at delivery from hospital records, and socio-economic indices and maternal age at menarche from questionnaires. For the baseline information, gestational age was calculated based on ultrasound measurements performed by an obstetrician combined with the last menstrual date. Birth weights had been measured by trained nurses in the delivery room. Mothers' education level and current household income were used as indicators of socioeconomic status. The education level was categorized into two levels: graduated from high school and graduated from college/university. Household income was classified into three groups by monthly income: $<3$ million KRW, 3.0-4.9 million KRW and $>5$ million KRW $(\mathrm{KRW}=$ South 
Korean Won). Data regarding maternal age at menarche were also collected as a proxy for genetic heritability ${ }^{(13)}$.

\section{Measurements}

Anthropometric data were collected by well-trained researchers when children aged 7-9 years visited the hospital. The height and weight of each child were measured with the child wearing light clothing and no shoes to the nearest $0.1 \mathrm{~cm}$ and $0.1 \mathrm{~kg}$, respectively, using a stadiometer and calibrated scale (DS-102; Dong Sahn Jenix, Co., Ltd, Seoul, Korea). Body fat mass as a measure of body composition was measured (Inbody 230; GE Healthcare, Madison, WI, USA). The BMI was calculated as weight divided by height squared $\left(\mathrm{kg} / \mathrm{m}^{2}\right)$. The height, weight and BMI were converted to age- and gender-specific $Z$-scores using the criteria from the 2007 Korean Children and Adolescents Growth Standards ${ }^{(14)}$. Age was treated in units of month. The weight $Z$-score change was calculated by subtracting the birth weight $Z$-score from the current weight $Z$-score, and was applied to the analysis as a continuous variable. Children with age- and gender-specific BMI $Z$-scores higher than the 85th percentile were classified as overweight, and obesity was determined based on current BMI. For comparison with other studies, overweight was defined using the criteria of the International Obesity Task Force ${ }^{(15)}$.

Subjects were instructed to fast for at least $8 \mathrm{~h}$ prior to providing a blood sample. We measured the levels of testosterone and oestradiol as indices of pubertal development. Testosterone was measured in boys using a commercial RIA kit (KIP1709; DIAsource ImmunoAssays S.A., Nivelles, Belgium); the minimum detection level in the assay was $0 \cdot 3 \mathrm{ng} / \mathrm{dl}$. The threshold level of testosterone considered to indicate entry into puberty $(250 \mathrm{ng} / \mathrm{dl})$ was established in a previous study ${ }^{(16)}$. The oestradiol level in the blood was measured in girls by a competitive electrochemiluminescence immunoassay method using an Elecsys E170 analyser (Elecsys Estradiol II; Roche Diagnostics GmbH, Mannheim, Germany); the minimum detection level was $5 \mathrm{pg} / \mathrm{ml}$. The oestradiol level considered to indicate entry into puberty was determined as $15 \mathrm{pg} / \mathrm{ml}$ based on a previous study ${ }^{(17)}$.

Insulin, insulin-like growth factor 1 (IGF-1) and leptin were also measured to investigate the relationships between their concentrations and pubertal development. Serum insulin was measured using an immunoradiometric assay kit (Biosource Europe, Nivelles, Belgium). The serum IGF-1 concentration was measured using a quantitative sandwich ELISA technique (Quantikine Human IGF-1 Immunoassay; R\&D Systems, Minneapolis, MN, USA). Serum leptin levels were measured using commercial kits (Millipore, Linco Research, St. Charles, MO, USA). All measurements had inter- and intraassay CV $<10 \%$.

\section{Assessment of early puberty}

Pubertal development was determined as clinicianreported Tanner stage assessments. Self-reported Tanner staging can be problematic, especially in differentiation between fat tissue and breast tissue in girls. Hence, assessments of anthropometric development were made following examination by clinicians. Early puberty was defined as Tanner stage 2 or higher ${ }^{(18,19)}$.

\section{Statistical analysis}

The results are given as means with standard deviations, and the categorical variables are expressed as the number of subjects and percentage. We compared the various features according to pubertal development status using the $t$ test for differences in means and the $\chi^{2}$ test for differences in frequency. The insulin and leptin concentrations were skewed, so the raw data were log-transformed. The postnatal growth from birth to follow-up according to pubertal development was evaluated based on weight, and changes were estimated using a mixed analysis. Repeated measurements within individuals were clustered according to the group, early puberty, or other categories. The model's fit accounted for variations causing different intercepts (random effect), with group, period (age) and the interaction between group and period (group $\times$ age) as fixed effects. To choose the working correlation, the model fit was assessed using the Bayesian information criterion. The results are presented as least-squared means (LSmeans) with standard errors using an unstructured covariance structure and are shown in Fig. 1.

We assessed the magnitude of the association between breast-feeding and early puberty by multiple logistic regression analysis with sequential adjustments for sets of covariates. Covariate selected were those with $P<0 \cdot 3$ on univariate analysis. Additionally, we considered other

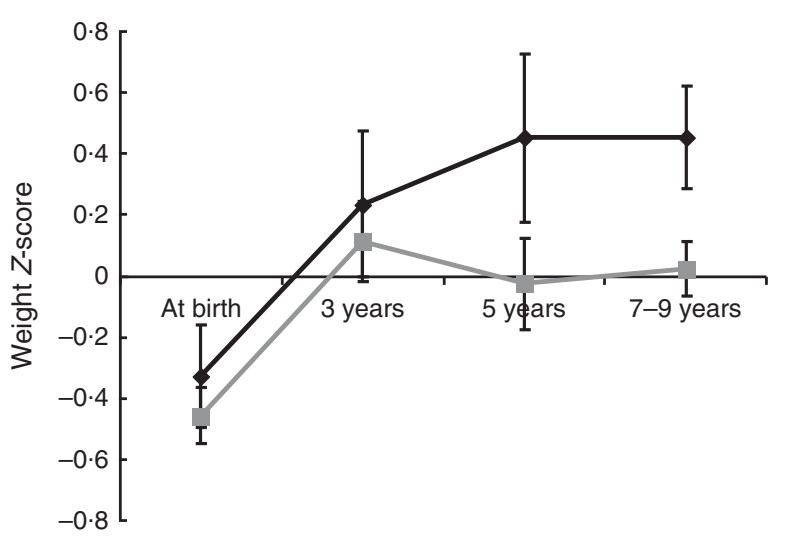

Fig. 1 The weight $Z$-score change from birth to current by puberty status ( - , early puberty; - - average puberty) among children aged 7-9 years, Ewha Birth \& Growth Cohort study, Republic of Korea. The weight Z-scores are standardized for age and sex and represent least-squared means with their standard errors represented by vertical bars at each time point, which were estimated using mixed analysis using unstructured covariance structure. The model's fit accounted for variations causing different intercepts (random effect), with the group, period (age), interactions between groups and period (group $\times$ age) being a fixed effect. At birth, $n 219$; age 3 years, $n$ 95; age 5 years, $n 72$; aged $7-9$ years, $n 219$ 
Table 1 Current anthropometric and endocrine characteristics by puberty status* among children aged 7-9 years ( $n$ 219), Ewha Birth \& Growth Cohort study, Republic of Korea

\begin{tabular}{|c|c|c|c|c|c|c|c|}
\hline & \multicolumn{2}{|c|}{ Total } & \multicolumn{2}{|c|}{ Early puberty $(n 50)$} & \multicolumn{2}{|c|}{ Average puberty ( $n$ 169) } & \multirow[b]{2}{*}{$P$ value } \\
\hline & $n$ or mean & $\%$ or SD & $n$ or mean & $\%$ or SD & $n$ or mean & $\%$ or SD & \\
\hline Sex (boys)‡ & 103 & $47 \cdot 0$ & 22 & $44 \cdot 0$ & 81 & 47.9 & 0.625 \\
\hline Age (years)§ & 8.00 & 0.86 & 8.40 & 0.78 & $7 \cdot 87$ & 0.85 & $<0.001$ \\
\hline \multicolumn{8}{|l|}{ Current anthropometric measurements } \\
\hline Sex- and age-specific height $Z$-score§ & 0.40 & 0.89 & 0.62 & 0.87 & 0.34 & 0.88 & 0.047 \\
\hline Sex- and age-specific weight $Z$ score $\S$ & 0.12 & 1.00 & 0.47 & 0.83 & 0.02 & 1.03 & 0.006 \\
\hline Sex- and age-specific BMI $Z$ score $\S$ & -0.14 & $1 \cdot 15$ & 0.22 & 0.92 & -0.24 & $1 \cdot 20$ & 0.012 \\
\hline Body fat mass $(\mathrm{kg}) \S$ & 6.98 & 3.86 & $8 \cdot 34$ & 3.97 & 6.58 & 3.75 & 0.004 \\
\hline Overweight ( $\geq 85$ th percentile of $\mathrm{BMI}) \ddagger, \|$ & 30 & $13 \cdot 7$ & 8 & $16 \cdot 0$ & 22 & $13 \cdot 0$ & 0.590 \\
\hline Overweight (IOTF criteria) $\ddagger, \uparrow$ & 50 & $22 \cdot 8$ & 16 & $32 \cdot 0$ & 34 & $20 \cdot 1$ & 0.079 \\
\hline Log insulin§ & 2.08 & 0.31 & $2 \cdot 16$ & 0.30 & 2.06 & 0.31 & 0.044 \\
\hline IGF-1 concentration in blood $(\mathrm{ng} / \mathrm{ml}) \S$ & 1.68 & 1.91 & 2.47 & $3 \cdot 16$ & 1.45 & 1.26 & 0.001 \\
\hline Log leptin§ & 1.70 & 0.64 & 1.77 & 0.70 & 1.68 & 0.62 & 0.435 \\
\hline
\end{tabular}

IOTF, International Obesity Task Force; IGF-1, insulin-like growth factor 1.

*Early puberty is defined as a subject who reached Tanner stage 2 or greater based on clinician-reported Tanner stage assessments.

$\dagger P$ value from $t$ test for differences in means and the $x^{2}$ test for differences in frequency.

\pm Values are $n$ and \%.

$\S$ Values are mean and SD.

IOverweight is defined as greater than the 85th percentile of age- and gender-specific BMI values, as referenced from the 2007 Korean Children and Adolescents Growth Standards ${ }^{(14)}$

ПOverweight is defined using the IOTF criteria $^{(15)}$.

Table 2 Distribution of measurements for pubertal development by puberty status* among children aged $7-9$ years $(n 219)$, Ewha Birth \& Growth Cohort study, Republic of Korea

\begin{tabular}{|c|c|c|c|c|c|}
\hline & \multicolumn{2}{|c|}{ Early puberty } & \multicolumn{2}{|c|}{ Average puberty } & \multirow[b]{2}{*}{$P$ value } \\
\hline & Median or $n$ & IQR or \% & Median or $n$ & IQR or \% & \\
\hline Boys ( $n$ 103) & \multicolumn{2}{|c|}{$n 22$} & \multicolumn{2}{|c|}{$n 81$} & \\
\hline Testosterone (ng/dl)‡ & 5.59 & $3 \cdot 37-8 \cdot 71$ & 5.45 & $3.24-8.00$ & 0.453 \\
\hline High testosterone level $(\geq 25 \mathrm{ng} / \mathrm{dl}) \S, \|$ & 1 & 4.6 & 2 & 2.5 & 0.518 \\
\hline Girls $(n 116)$ & \multicolumn{2}{|c|}{$n 28$} & \multicolumn{2}{|c|}{$n 88$} & \\
\hline Oestradiol (pg/ml)‡ & $15 \cdot 00$ & $7 \cdot 40-37.75$ & 7.45 & $4.90-10 \cdot 65$ & $<0.001$ \\
\hline High oestradiol level $(\geq 15 \mathrm{pg} / \mathrm{ml}) \S, \mathbb{\Upsilon}$ & 15 & 53.6 & 8 & 9.1 & $<0.0001$ \\
\hline
\end{tabular}

IQR, interquartile range.

*Early puberty is defined as a subject who reached Tanner stage 2 or greater based on clinician-reported Tanner stage assessments.

$\dagger P$ value obtained from Wilcoxon rank-sum test for non-parametric value and Fisher's exact test for differences in frequency.

$\ddagger$ Values are median and IQR.

$\S$ Values are $n$ and \%.

IIThe cut-off for testosterone refers to the study by Potau et al. ${ }^{(16)}$.

IThe cut-off for oestradiol refers to the study by Prété et al. ${ }^{(17)}$

factors, such as covariates that have been shown to be related to pubertal development. Covariates, classified considering age and gender, included maternal effects (maternal age at delivery and maternal age at menarche) and birth effects (birth weight ${ }^{(14)}$, gestational age and postnatal growth effect $\left.{ }^{(20)}\right)$. All analyses were two-tailed and $P<0.05$ was taken to indicate statistical significance. All statistical analyses were performed using the SAS statistical software package version 9.1.

\section{Results}

Fifty (22.8\%) of the 219 children included in the present study were in early puberty, with the proportion being slightly higher for girls (28/116 girls, $24 \cdot 1 \%)$ than boys (22/103 boys, $21.4 \% ; \quad P=0.625)$. The subjects who experienced early puberty were significantly taller, weighed more and had a higher BMI than those whose pubertal development was average. However, the distribution of overweight children did not differ according to pubertal development. Using the International Obesity Task Force criteria, the proportion of overweight subjects was higher in the early puberty group. However, this finding showed only borderline significance. The early puberty group had elevated levels of log-transformed insulin and IGF-1 compared with the average puberty group (Table 1).

Of the twenty-eight girls in the early puberty group, fifteen $(53.4 \%)$ had higher levels of oestradiol, whereas very few boys in the early puberty group had high testosterone levels; overall, the early maturing boys showed similar mean testosterone level to boys in the average puberty group (Table 2 ). 
Table 3 Effect of potential risk factors on puberty status among children aged 7-9 years ( $n$ 219), Ewha Birth \& Growth Cohort study, Republic of Korea

\begin{tabular}{|c|c|c|c|c|c|}
\hline & \multicolumn{2}{|c|}{ Early puberty $(n 50)$} & \multicolumn{2}{|c|}{ Average puberty ( $n$ 169) } & \multirow[b]{2}{*}{$P$ value } \\
\hline & $n$, mean or median & $\%$, SD or IQR & $n$, mean or median & $\%$, SD or IQR & \\
\hline \multicolumn{6}{|l|}{ Main effect } \\
\hline Breast-feeding for $\geq 6$ months (yes) ${ }^{\star}, \dagger$ & 15 & $30 \cdot 0$ & 98 & $58 \cdot 0$ & $<0.001$ \\
\hline \multicolumn{6}{|l|}{ Factors of socio-economic status and maternal effect } \\
\hline Maternal age at delivery (years) $\ddagger$ & 31.66 & 2.87 & $31 \cdot 37$ & 3.85 & 0.630 \\
\hline Maternal age at menarche (years)§ & 13.5 & $12 \cdot 5-14.5$ & $14 \cdot 0$ & $13 \cdot 0-15 \cdot 0$ & 0.876 \\
\hline \multicolumn{6}{|l|}{ Maternal education†,॥ } \\
\hline Graduated high school & 11 & $22 \cdot 0$ & 37 & $22 \cdot 0$ & 0.997 \\
\hline Above college or university & 39 & 78.0 & 131 & 78.0 & \\
\hline \multicolumn{6}{|l|}{ Household incomet,\| } \\
\hline$<3$ million $\mathrm{KRW}$ & 6 & $12 \cdot 0$ & 28 & $16 \cdot 7$ & 0.657 \\
\hline 3-5 million KRW & 24 & 48.0 & 71 & $42 \cdot 3$ & \\
\hline$\geq 5$ million KRW & 20 & $40 \cdot 0$ & 69 & $41 \cdot 1$ & \\
\hline \multicolumn{6}{|l|}{ Factors of birth outcomes and growth pattern } \\
\hline Birth weight $(\mathrm{kg}) \ddagger$ & $3 \cdot 24$ & 0.45 & $3 \cdot 21$ & 0.54 & 0.732 \\
\hline Gestational age (weeks)§ & 39.2 & $38 \cdot 3-40 \cdot 1$ & 39.1 & $38 \cdot 1-40 \cdot 2$ & 0.621 \\
\hline Change in weight $Z$-score from birth to follow-up $\ddagger$ & 0.79 & 1.43 & 0.47 & 1.66 & 0.214 \\
\hline
\end{tabular}

IQR, interquartile range; KRW, South Korean Won.

*We classified the subjects into two groups: those who breast-fed for more than 6 months and those who did not.

†Values are $n$ and \%; $P$ value obtained from $X^{2}$ test for differences in frequency.

$\ddagger$ Values are mean and SD; $P$ value obtained from $t$ test for differences in means.

$\S$ Values are median and IQR; $P$ value of non-parametric analysis was conducted using the Wilcoxon rank-sum test.

IN Number of subjects varies due to missing data.

Among the total of 171 children (78.1\%) who were breast-fed, $66 \cdot 1 \%$ ( $n$ 113) were breast-fed for 6 months or more. More than half of the children in the average puberty group were breast-fed for at least 6 months, whereas this was true for only about $30 \%$ of those in the early puberty group. The maternal age at menarche with early pubertal children was younger than that of mothers whose children were of average age at puberty, but this finding was not significant. Maternal age at delivery, maternal education level, household income, birth weight and gestational age were not significantly different between the two groups (Table 3).

The change in weight $Z$-score from birth until follow-up in children with early puberty was slightly higher than that for children with average puberty, although the difference was not statistically significant (weight $Z$-score change $=$ 0.79 (SD 1.43) v. 0.47 (SD 1.66), respectively, $P=0.21$ ). We analysed the group changes in weight $Z$-scores over time; the results indicated that the slope of weight change for children who experienced early puberty showed a gradual and persistent increase in weight $Z$-score, whereas for the average puberty group, the slope tended towards zero with increasing age. The effect of group was marginally insignificant $(P=0 \cdot 06$; Fig. 1$)$. When we assessed whether breast-feeding influenced weight change, the change in weight $Z$-score from birth until follow-up in children who were breast-fed for 6 months or more was significantly lower compared with that for children who were not (weight $Z$-score change $=0.32(\mathrm{SD} 1.59) v .0 .77$ (sD $1 \cdot 61$ ), respectively, $P=0 \cdot 04$ ).

The associations of breast-feeding and early pubertal development with sequential adjustment for sets of covariates were analysed (Table 4). Breast-feeding for at least 6 months showed a strong preventive association against early puberty. The association remained even after accounting for maternal features (model 2) and for additional birth features and postnatal growth (model 3; $\mathrm{OR}=0.40 ; 95 \% \mathrm{CI} 0 \cdot 19,0.83$ and $\mathrm{OR}=0 \cdot 35 ; 95 \% \mathrm{CI} 0 \cdot 16$, $0 \cdot 75$, respectively).

In the final model (model 3), even after reclassifying the children into three groups, i.e. breast-fed for $\geq 6$ months, breast-fed for $<6$ months and not breast-fed at all, with the 'not breast-fed at all' group as the reference, the protective association of breast-feeding for 6 months or more and early pubertal development remained $(\mathrm{OR}=0 \cdot 27$; $95 \% \mathrm{CI}$ $0 \cdot 11,0 \cdot 66$ ). Additionally, breast-feeding for $<6$ months also showed a protective association against early pubertal development, but the $95 \%$ confidence interval included $1.0(\mathrm{OR}=0.61 ; 95 \% \mathrm{CI} 0.24,1.55)$, indicating that the association was not significant.

\section{Discussion}

A major finding of the present study was that nutrition during infancy is associated with pubertal development: breast-feeding for 6 months or more showed a distinct preventive association against early pubertal development. Moreover, we found a tendency towards greater weight gain with greater current height, weight and BMI status in children with early puberty, although the proportion of overweight children did not differ from that among children who entered puberty at an average age. Our results suggest that a reasonable duration of 
Table 4 Model specifications for the impact of breast-feeding for more than 6 months on early pubertal development, with sequential adjustments for sets of covariates, among children aged 7-9 years ( $n$ 219), Ewha Birth \& Growth Cohort study, Republic of Korea

\begin{tabular}{|c|c|c|c|c|c|c|}
\hline & \multicolumn{2}{|c|}{$\begin{array}{l}\text { Model } 1 \text { (breast- } \\
\text { feeding effect) }\end{array}$} & \multicolumn{2}{|c|}{$\begin{array}{l}\text { Model } 2 \text { (model } 1+ \\
\text { maternal features) }\end{array}$} & \multicolumn{2}{|c|}{$\begin{array}{l}\text { Model } 3 \text { (model } 2+\text { birth } \\
\text { features and postnatal growth) }\end{array}$} \\
\hline & OR & $95 \% \mathrm{Cl}$ & OR & $95 \% \mathrm{Cl}$ & OR & $95 \% \mathrm{Cl}$ \\
\hline Breast-feeding for $\geq 6$ months (yes)* & 0.37 & $0.18,0.74$ & 0.40 & $0.19,0.83$ & 0.35 & $0.16,0.75$ \\
\hline Maternal age at delivery (years) & & & 1.04 & $0.94,1.14$ & 1.04 & $0.94,1.15$ \\
\hline Maternal age at menarche (years) & & & 0.90 & $0.71,1 \cdot 14$ & 0.93 & $0.73,1 \cdot 18$ \\
\hline Birth weight $(\mathrm{kg})$ & & & & & 2.04 & $0.61,6 \cdot 86$ \\
\hline Gestational age (weeks) & & & & & $1 \cdot 28$ & $0.98,1.68$ \\
\hline Change in weight $Z$-score from birth to follow-up & & & & & 1.49 & $1.04,2 \cdot 14$ \\
\hline
\end{tabular}

Results present adjusted odds ratio and $95 \%$ confidence intervals, which were derived from multiple logistic regression analysis. All models basically adjusted for sex and age.

*We classified the subjects into two groups: those who breast-fed for more than 6 months and those who did not.

breast-feeding intervention has a protective association against early pubertal development.

Puberty is a complex, coordinated biological process that leads to the eventual acquisition of reproductive capability. Although the onset of puberty is a natural event, as stated above, its initial timing can be influenced by multiple factors. Some studies have suggested that early puberty is associated with chronic diseases such as polycystic ovary syndrome, breast cancer and CVD in later life ${ }^{(3,4)}$. Therefore, the decrease in average age of onset of puberty over the past several decades has become a public health issue worldwide. The key to resolving this issue is to identify modifiable risk factors for prevention of early puberty. Infancy has been proposed as a critical period for determining pubertal timing and several authors considered infant feeding as a potential modifiable risk factor $^{(2,5,6)}$. However, there was a lack of evidence to support this recommendation due to heterogeneity among studies with respect to definitions, method of puberty assessment and study subjects, although breast-feeding had clear advantages.

Evidence collected over the last few decades suggests that differences in diet during infancy may be partially responsible for differences in pubertal development. According to the Cebu Longitudinal Health and Nutrition Survey, which involved population-based surveys, the risk of earlier menarche decreased by $6 \%$ with each 1 -month increase in the duration of breast-feeding (hazard ratio $=$ 0.94; $95 \%$ CI 0.90, 0.98). They also suggested that exclusive breast-feeding is more important than the duration of breast-feeding ${ }^{(5)}$. However, they did not consider covariates such as postnatal growth. Breast-feeding is associated with weight gain during early life, which itself is a possible candidate factor for untimely puberty development. Morris et al. reported the determinants of age at menarche based on the Breakthrough Generations Study, but the effect of breast-feeding was difficult to fully explain due to the large volume of missing responses in the survey regarding breastfeeding ${ }^{(21)}$. Moreover, these two studies were conducted primarily on girls. Although our study was relatively small in size compared with the two studies mentioned above, we found a marked preventive association against early pubertal development in children who were breast-fed for at least 6 months even after controlling for relevant covariates $(\mathrm{OR}=0.35 ; 95 \% \mathrm{CI} 0 \cdot 16,0 \cdot 75)$.

Although the mechanisms underlying the relationship between breast-feeding and initiation of puberty are still unclear, several studies have suggested that it may be explained by weight gain during childhood. One Danish cohort study reported that breast-feeding delayed weight gain in early life and that children who were breast-fed for more than 40 weeks had lower weight than those who were breast-fed for less than 20 weeks ${ }^{(22)}$. Kwak et al. reported similar results ${ }^{(23)}$. Our findings were consistent with the above studies. Some studies indicated that weight gain during the first 2 years of life predicted the subsequent timing of puberty onset ${ }^{(8)}$. Growth during infancy is largely regulated by nutrition. Hence, it has been suggested that nutrition during infancy may program growth and development. When considering weight at 3 years instead of that at 2 years due to lack of data, the results of the present study were similar to previous studies. Children who underwent puberty early showed accelerated weight gain in the first 3 years of life. Moreover, higher postnatal change in weight $Z$-score was associated with increased risk of early puberty in the present study. In sub-analyses, the protective association of breast-feeding and early pubertal development remained even after adjusting for the change in weight $Z$-score in the first 3 years of life ( $n$ 95) instead of those from birth to the present, although the $P$ value for weight change for the first 3 years of life was not significant (data not shown).

Breast-feeding can contribute to appetite programming, which is explained by leptin hormone regulation ${ }^{(24,25)}$. An animal study suggested that appetite may be upregulated in formula-fed babies, which may lead to obesity ${ }^{(26)}$. Additionally, leptin secreted from adipose tissue is also involved in the activation of central gonadotrophin secretion. However, the evidence remains insufficient to reach definitive conclusions. In the present study, the log-leptin level was higher in the early puberty group compared with the average puberty group, although the 
difference was not significant (1.77 (SD 0.70$) \quad v .1 .68$ (sD 0.62), respectively, $P>0.05$ ). However, it was difficult to directly determine the relationship between leptin and the pubertal process because leptin levels have been shown to be tightly linked with body weight $(r=0.61, P<0.0001)$ and body fat mass $(r=0.75, P<0.0001)^{(27)}$. Alternatively, insulin or IGF-1 may account for the differences in the age of puberty. High insulin level is related to the level of sex hormone-binding globulin and may therefore upregulate the bioavailability of sex steroids. Additionally, an increase in IGF-1 due to rapid weight gain was suggested to mediate pubertal development, although the results were not conclusive $^{(28)}$. These potential mechanisms are consistent with our findings. The IGF-1 hormone levels in subjects with early puberty were higher than those in the average puberty group. Additionally, the average log-transformed insulin level was also significantly different between the two groups (2.16 (SD 0.30) v. 2.06 (SD 0.34), respectively, $P=0 \cdot 04$ ). Unfortunately, our understanding of how breastfeeding affects physiological and endocrine changes and how long these effects persist is still limited. Further studies are needed to verify the associations through longitudinal observations. Prior obesity has also been suggested as a risk factor for early puberty. In the present study, children with early puberty had higher weight, BMI and body fat mass, although the association between early puberty and overweight was not significant. This finding is probably reflective of inadequate statistical power caused by the small number of subjects included in the study.

Despite considering potential confounding factors, including the maternal age at menarche as a proxy for genetic heritability, none of the factors other than change in weight was a significant predictor of early puberty. Although the average age of children with early pubertal development was higher than that in the other group, the protective association of breast-feeding for 6 months or more and early pubertal development was still significant, even after considering potential confounding factors such as age.

There were several limitations in the present study. First, we used a self-reported questionnaire about breastfeeding and did not obtain detailed information about breast-feeding practices, which made it difficult to define exclusive breast-feeding. However, the rate of exclusive breast-feeding in Korea has greatly declined from 1994 to 2012. Furthermore, the proportion of children who were breast-fed for 6 months (even if not exclusively) was similar to that reported in the Korea National Health and Nutrition Examination Survey in 2001, 2005 and 2007 (49.5\%, 49.6\%, and $55.8 \%$, respectively) ${ }^{(29)}$. Some uncertainty may have influenced the results, but the reliability of the questions on breast-feeding duration was high. Second, the study recruited subjects born in one hospital and this limited sample makes it difficult to generalize to other populations. The lack of statistical power due to the small sample size also limited the generalizability of the results. Limited data regarding growth pattern were available due to unavoidable data loss in the follow-up process. Finally, residual confounding factors that we did not measure may have affected the association between breast-feeding and early puberty.

Nevertheless, our study had advantages over previous investigations in that we applied a reasonable criterion for puberty assessment and did not limit our subjects to a single gender. Moreover, clinicians assessed pubertal development to avoid the potential errors that may result from self-report. The study was also worthwhile because there have been few such studies in the Asia-Pacific region. These results were derived from a well-timed assessment that specifically considered the age of the subjects. Previous studies on the relationship between breast-feeding and pubertal development were conducted through retrospective collection of event-history data. An additional strength of our study was that we controlled for potential confounding factors. The nutrition status in early life is solely dependent on the mother and nutrition status can be modified.

The relationship between breast-feeding and pubertal development is thought to involve a stepwise causal pathway from nutrition in infancy to weight status and finally to pubertal development. Although our findings did not clearly identify a plausible mechanism, we found an independent preventive association of breast-feeding for 6 months or more and early pubertal development even after adjusting for relevant covariates. Therefore, although further studies are required, we suggest that breast-feeding is a modifiable risk factor that can contribute to improvement of child health, helping to prevent both extremes of the growth pattern. Intervention must be started early in life to prevent early pubertal development.

\section{Acknowledgements}

Financial support: This work was supported by National Research Foundation of Korea Grant funded by the Korean Government (2010-0026225) and the Ewha Womans University Research Grant of 2014. The funders had no role in the design, analysis or writing of this article. Conflict of interest: None. Authorship: H.P. has overall responsibility for this paper. H.A.L. wrote the paper with statistical analysis and data collection; E.A.P., S.J.C., H.S.K. and Y.J.K. helped with study participant recruitment and data collection; E.A.P., S.J.C., H.S.K., Y.J.K., H.L., H.S.G., Y.S.H., E.H.H. and H.P. gave advice for writing the paper; Y.J.K. and Y.S.H. contributed to interpretation of data. Ethics of buman subject participation: The study protocol was approved by the Institutional Review Board of Ewha Womans University Hospital.

\section{References}

1. Cho GJ, Park HT, Shin JH et al. (2010) Age at menarche in a Korean population: secular trends and influencing factors. Eur J Pediatr 169, 89-94. 
2. Cesario SK \& Hughes LA (2007) Precocious puberty: a comprehensive review of literature. I Obstet Gynecol Neonatal Nurs 36, 263-274.

3. Petridou E, Syrigou E, Toupadaki N et al. (1996) Determinants of age at menarche as early life predictors of breast cancer risk. Int J Cancer 68, 193-198.

4. Lakshman R, Forouhi NG, Sharp SJ et al. (2009) Early age at menarche associated with cardiovascular disease and mortality. J Clin Endocrinol Metab 94, 4953-4960.

5. Al-Sahab B, Adair L, Hamadeh MJ et al. (2011) Impact of breast feeding duration on age at menarche. Am J Epidemiol 173, 971-977.

6. Novotny R, Daida YG, Grove JS et al. (2003) Formula feeding in infancy is associated with adolescent body fat and earlier menarche. Cell Mol Biol (Noisy-le-grand) 49, 1289-1293.

7. World Health Organization (2001) Global Strategy for Infant and Young Child Feeding. The Optimal Duration of Exclusive Breast Feeding. Geneva: WHO; available at http:// apps.who.int/gb/archive/pdf_files/WHA54/ea54id4.pdf

8. Karaolis-Danckert N, Buyken AE, Sonntag A et al. (2009) Birth and early life influences on the timing of puberty onset: results from the DONALD (DOrtmund Nutritional and Anthropometric Longitudinally Designed) Study. Am J Clin Nutr 90, 1559-1565.

9. Blell M, Pollard TM \& Pearce MS (2008) Predictors of age at menarche in the Newcastle thousand families study. J Biosoc Sci 40, 563-575.

10. Moisan J, Meyer F \& Gingras S (1990) A nested case-control study of the correlates of early menarche. Am J Epidemiol 132, 953-961.

11. Kwok MK, Leung GM, Lam TH et al. (2012) Breastfeeding, childhood milk consumption, and onset of puberty. Pediatrics 130, e631-e639.

12. Min JW, Kong KA, Park BH et al. (2007) Effect of postnatal catch-up growth on blood pressure in children at 3 years of age. J Hum Hypertens 21, 868-874.

13. Ong KK, Northstone K, Wells JC et al. (2007) Earlier maternal age at menarche predicts rapid infancy growth and childhood obesity. PLoS Med 4, e132.

14. Korea Center for Disease Control and Prevention, The Korean Pediatric Society, The Committee for the Development of Growth Standard for Korean Children and Adolescents (2008) 2007 Korean Children and Adolescents Growth Standard (commentary for the development of 2007 growth chart). Seoul: Division of Chronic Disease Surveillance; available at http://www.cdc.go.kr (in Korean).
15. Cole TJ, Bellizzi MC, Flegal KM et al. (2000) Establishing a standard definition for child overweight and obesity worldwide: international survey. BMJ 320, 1240-1243.

16. Potau N, Ibáñez L, Sentis M et al. (1999) Sexual dimorphism in the maturation of the pituitary-gonadal axis, assessed by GnRH agonist challenge. Eur J Endocrinol 141, 27-34.

17. Prété G, Couto-Silva AC, Trivin C et al. (2008) Idiopathic central precocious puberty in girls: presentation factors. BMC Pediatr 8, 27.

18. Marshall WA \& Tanner JM (1969) Variations in pattern of pubertal changes in girls. Arch Dis Child 44, 291-303.

19. Marshall WA \& Tanner JM (1970) Variations in the pattern of pubertal changes in boys. Arch Dis Child 45, 13-23.

20. dos Santos Silva I, De Stavola BL, Mann V et al. (2002) Prenatal factors, childhood growth trajectories and age at menarche. Int J Epidemiol 31, 405-412.

21. Morris DH, Jones ME, Schoemaker MJ et al. (2010) Determinants of age at menarche in the UK: analyses from the Breakthrough Generations Study. Br J Cancer 103, 1760-1764.

22. Baker JL, Michaelsen KF, Rasmussen KM et al. (2004) Maternal prepregnant body mass index, duration of breast feeding, and timing of complementary food introduction are associated with infant weight gain. Am J Clin Nutr 80, 1579-1588.

23. Kwak JY, Park JY, Lee HJ et al. (2005) Growth patterns of breast fed and formula fed infants. Korean J Pediatr $\mathbf{4 8}$, 1055-1060 (in Korean).

24. Singhal A \& Lanigan J (2007) Breast feeding, early growth and later obesity. Obes Rev 8, Suppl. 1, 51-54.

25. Singhal A, Farooqi IS, O'Rahilly S et al. (2002) Early nutrition and leptin concentrations in later life. Am J Clin Nutr 75, 993-999.

26. Ozanne SE \& Hales CN (2004) Lifespan: catch-up growth and obesity in male mice. Nature 427, 411-412.

27. Garcia-Mayor RV, Andrade MA, Rios M et al. (1997) Serum leptin levels in normal children: relationship to age, gender, body mass index, pituitary-gonadal hormones, and pubertal stage. J Clin Endocrinol Metab 82, 2849-2855.

28. Dunger DB, Ahmed ML \& Ong KK (2006) Early and late weight gain and the timing of puberty. Mol Cell Endocrinol 254-255, 140-145.

29. Chung SH, Kim HR, Choi YS et al. (2013) Trends of breastfeeding rate in Korea (1994-2012): comparison with OECD and other countries. I Korean Med Sci 28, $1573-1580$. 